\title{
Nouvelle poétique de l'intimité chez Pierre Reverdy
}

\author{
Marie-Noëlle BROGLY \\ University of Saint Andrews, School of Modern Languages, Department of French \\ mb744@st-andrews.ac.uk
}

Recibido: $30 / 10 / 2012$

Aceptado: 28/01/2013

\begin{abstract}
Résumé
L'écriture de l'intimité, telle qu'elle se comprend au XIXème siècle, s'appuie généralement sur une mise en scène plus ou moins dramatique de la subjectivité souffrante du poète offerte au lecteur. Exhiber son être profond est pour le poète le moyen de susciter une intimité, de nature cette fois littéraire, avec le lecteur. Pierre Reverdy vient bouleverser cet équilibre en proposant d'émouvoir le lecteur tout en écrivant une poésie sans sujet, portée par la voix, en déplaçant le lyrisme de la subjectivité du poète au choc des images. Reverdy parvient ainsi à créer une nouvelle intimité, en faisant prendre en charge la voix lyrique de ses poèmes par le lecteur, poussé à puiser en lui-même de quoi remplir les interlignes du poème. Ce faisant, le poète ouvre la voie à une poésie mettant cette fois l'intimité du lecteur au premier plan, et dont le but ultime est déplacé de l'expression de soi à celle de l'indicible que représente pour nous notre être-au-monde.
\end{abstract}

Mots clefs: Reverdy, intimité, subjectivité, lyrisme, voix, Collot, Rodriguez.

\section{Nueva poética de la intimidad en la obra de Pierre Reverdy}

\begin{abstract}
Resumen
En el siglo XIX, la intimidad, para el poeta, suele significar la representación en el verso de su proprio sufrimiento, que ofrece al lector, con un grado variable de drama. La exposición de su intimidad personal es una forma de crear otro tipo de intimidad de naturaleza literaria entre el poeta y el lector. Pierre Reverdy rompe este equilibrio, emocionando al lector en su intimidad, cuando traslada el origen del lirismo del ser del poeta al choque creado por las asociaciones de palabras en la imagen poética. Reverdy da luz a una nueva forma de intimidad, en la cual el lector, llevado a descubrir lo que yace escondido entre las líneas del poema, se transforma en responsable de la voz lírica. Así, el poeta da voz a una poesía en la cual se destaca la intimidad del lector, y cuyo objetivo supremo cambia de la expresión del ser lo indecible de nuestro estar-en-el-mundo.
\end{abstract}

Palabras clave: Reverdy, intimidad, subjectividad, lirismo, voz, Collot, Rodriguez.

\section{New poetics of Intimacy in the work of Pierre Reverdy}

\begin{abstract}
In the nineteenth century, intimacy, for the poet, usually signifies staging the suffering of his own self in his verse, with a variable degree of drama, and offering it to the reader. The exhibition of personal intimacy is a way of creating another form of intimacy, this time of a literary nature, between poet and reader. Pierre Reverdy shattered this system when - while proposing to touch the reader emotionally - he wrote poems without a subject, whose enunciation was carried out by a poetic voice, and when he moved the source of lyricism from the self to the shock created by word associations in the
\end{abstract}


poetic image. A new form of intimacy is thus created, in which the reader has to take responsibility for the lyrical voice, and is then required to look into himself to till out what lies between the lines of the poem. By doing so, the poet opens a door for a poetry featuring the intimacy of the reader, a poetry whose ultimate goal shifts from the expression of the self to the expression of the unsayable, our state of being in the world.

Keywords: Reverdy, intimacy, subjectivity, lyricism, voice, Collot, Rodriguez.

\section{Referencia normalizada}

Brogly, M.-N. (2013). "Nouvelle poétique de l'intimité chez Pierre Reverdy”. Thélème, Vol. 28,61-71.

S'il est largement accepté aujourd'hui, depuis le Contre Sainte-Beuve que le « je » de l'auteur ne renvoie pas nécessairement à sa personne privée, ces réflexions concernent largement la prose. La poésie est restée à l'écart de ces questions ; Hugo, Verlaine, Rimbaud ou même Apollinaire n'ont jamais semblé avoir de problème à puiser abondamment dans leur vie personnelle pour nourrir leur poésie. Chacun ouvre dans ces vers une porte différente sur son intimité dans le but d'émouvoir son lecteur; Hugo le fait en mêlant clairement sa vie à ses vers, Verlaine suggère des situations ou des sentiments que l'étude de sa biographie permet parfois rétrospectivement d'éclairer. Dans les deux cas, il s'agit de mettre à jour dans ses vers ses sentiments les plus personnels et de les partager avec le lecteur, mettant par là une double intimité en jeu : celle révélée dans le poème et celle qui s'en suit entre poète et lecteur. Le commentaire de Victor Hugo à cet égard semble parfaitement résumer la base de cette relation:

Nul n'a l'honneur d'avoir une vie qui soit à lui. Ma vie est la vôtre, votre vie est la mienne, vous vivez ce que je vis ; la destinée est une. Prenez donc ce miroir et regardez-vous-y. (Hugo, 1967 : 28, Préface).

Pierre Reverdy ne semble pas déroger à la tradition qui articule intimité du poète et émotion du lecteur, lorsqu'il écrit à propos de la mission du poète :

Car, si ce qui importe surtout c'est ce qu'il peut avoir à dire pour exprimer sa personnalité la plus intime, ce qui importe autant, au moins, ce sera la façon de le dire. En effet, pour si étrange que cela puisse paraître, ce sera la façon particulière de dire une chose très simple et très commune qui ira la porter au plus secret, au plus caché, au plus intime d'un autre et produira le choc (Reverdy, 2010b : 1287).

Néanmoins, si la poésie de Reverdy vise la communication d'intime à intime, elle le fait de manière différente de ce qui existait auparavant: elle n'offre pas de détail biographique et interdit toute identification entre le «je » présent dans ses vers et le poète, en réduisant l'instance en charge de l'énonciation à une simple voix sans subjectivité. Reverdy propose d'effectuer ce qu'il appelle « la soudure d'âme à âme dans le choc-poésie » (Reverdy, 2010b : 1291) en réduisant l'expression à son 
minimum vital, posant ainsi les bases d'une nouvelle façon d'écrire l'intimité au XXème siècle.

Stanislas Fumet, en appendice de l'édition de 1968 du Gant de crin raconte une anecdote selon laquelle la librairie Plon demanda différentes informations à Reverdy, dans le cadre de la publicité entourant la première publication dudit ouvrage. Reverdy à la question des «renseignements biographiques» choisit de répondre « sans intérêt », ce que Fumet commente ainsi :

Tout Reverdy est dans ce dernier mot : il rejette par là une biographie qu'il ne se jugeait pas tenu de dévoiler au public. On sait que dans son œuvre il ne se raconte jamais, alors que l'homme y est partout (Reverdy, 1968 : 179).

Fumet résume en un paradoxe la poésie reverdienne : dire l'homme en ignorant le « je », créer une communauté entre lecteurs et avec le poète, en laissant l'individu de côté pour se concentrer sur ce qu'il y a plus radicalement humain en chacun : l'émotion. C'est grâce à elle que le poète entend toucher le lecteur, non en tâchant, à travers une anecdote, de susciter son empathie par un processus d'identification, mais en dénudant sa poésie de tout ce qui relève du particulier pour se concentrer sur l'émotion pour ainsi dire brute, offerte au lecteur par la médiation de l'image et du lyrisme. Il ne s'agit pas là cependant du lyrisme tel qu'on le connaît à l'époque où le poète commence à écrire ; Reverdy le condamne, pour mieux le faire renaître par la suite :

Je ne crois pas au lyrisme par quoi on a pu autrefois chercher à émouvoir, au bercement du vent sortant d'un tuyau creux, au ronflement sonore, aux effets de la voix.

Je ne crois plus possible l'exaltation échevelée, la déclamation pénible jaillie d'une poitrine oppressée. Tout le mal que se donnent les grands acteurs pour gonfler inutilement de pauvres vers. Enfin, je ne crois pas qu'on puisse raisonnablement se proposer d'emporter l'âme d'un lecteur, d'un auditeur, dans un grand courant d'air (Reverdy, 2010a : 576-77).

Pour Reverdy, le ressort de l'émotion poétique ne peut plus passer par le lyrisme compris comme une posture artificielle digne d'un acteur de théâtre. Le poète ne peut plus émouvoir en se mettant en scène dans une posture dramatique, comme cela put être le cas à l'époque romantique, tel que le sous-entend le poète, tout en cherchant à en dégager le lyrisme. Aussi, pour prendre acte de ce divorce qu'il essaie d'effectuer, Reverdy bannit les détails biographiques de son œuvre. Collot parle à cet égard de « dépassement de l'anecdote » (Collot, 1997 : 211) pour qualifier la position de Reverdy quant à ce qui peut faire l'objet d'un poème.

Ce refus de situer ses poèmes dans l'histoire d'une subjectivité s'effectue à travers différents choix stylistiques, linguistiques et lexicaux pour Reverdy, que l'étude de «Et Là » du recueil Les Ardoises du toit, représentatif de la manière du poète, permettra de mieux comprendre (Reverdy, 2010a : 229) :

Quelqu'un parle et je suis debout

Je vais partir là-bas à l'autre bout

Les arbres pleurent

Parce qu'au loin d'autres choses meurent 
Maintenant la tête a tout pris

\author{
Mais je ne t'ai pas encore compris \\ Je marche sur tes pas sans savoir qui je suis \\ Il faut passer par une porte où personne n'attend \\ Pour un impossible repos \\ Tout s'écarte et montre le dos \\ Un peu de vide reste autour \\ Et pour revivre d'anciens jours \\ Une âme détachée s'amuse \\ Et traîne encore un corps qui s'use \\ Le dernier temps d'une mesure \\ Plus tenace et plus déchirant \\ Un chagrin musical murmure
}

Ce poème se signale d'emblée par le vague de sa référence à plusieurs niveaux : spatial, temporel et personnel. Le poème évoque un «là-bas », un " au-delà », un « autre bout», une « une porte où personne n'attend». Le ou les lieux auxquels ces termes peuvent renvoyer ne sont jamais identifiés dans le poème, au mépris, pour la moitié d'entres-eux, de leur signification réelle : «là-bas » ne fonctionne que par opposition à un ici, et " autre » suppose un élément originaire, un départ peut-être, qui n'est jamais mentionné. Au niveau temporel, l'ensemble du poème est écrit dans un présent indistinct qui peut avoir valeur de vérité générale comme si la scène se répétait à l'infini, ou donner au récit un ton solennel et en faire une expérience unique. Les deux lectures sont possibles et concurrentes. Un futur est annoncé «je vais partir » sans être détaillé, et un passé est évoqué « d'anciens jours », peut-être situé dans une histoire, personnelle ou collective. Deux passés composés sont présents : «la tête a tout pris» et «je ne t'ai pas encore compris », mais ils n'orientent pas pour autant le texte vers le passé dans la mesure où ils servent plutôt à établir un constat visant à expliquer la situation actuelle. Au niveau personnel, les pronoms « je » et «tu» sont représentés dans le poème, « je » est en charge de l'énonciation, mais aucun détail dans le poème ne permet de l'identifier ou de lui prêter une forme de subjectivité (un caractère, des sentiments, des émotions propres), en écho au second vers de la deuxième strophe où le « je » lui-même reconnaît ne pas savoir qui il est. Le «tu » n'est présent qu'en position d'objet, de récipiendaire - là encore non identifié - de cette parole. L'absence de détail qui, paradoxalement, le constitue peut en faire tour à tour: une personne que seule le poète connaîtrait, une allégorie de quelque chose (l'inspiration, la mort...), l'autre absolu, ou encore le lecteur. Il est remarquable dans ce poème que le lecteur a le choix de prendre en charge l'énonciation - et d'être derrière le « je $»$, ou de penser que le poète s'adresse à lui —et de s'identifier au «tu ». Les autres, potentiellement représentés par " quelqu'un » (ligne 1) et " personne » (ligne 8), ne sont pas plus identifiés, ce qui accentue l'impression émanant de la lecture que le « je » est bel est bien seul au monde. Le vague de la référence montre déjà qu'une part importante de l'interprétation de ce poème est laissée au lecteur. 
Un autre élément concourant à l'obscurité relative de ce poème est l'usage que Reverdy fait des articles définis et indéfinis. Les articles indéfinis dominent clairement le poème : « une porte », « un repos », «d'anciens jours ", « une âme », « un corps », « une mesure », « un chagrin musical ». Dans aucun des cas il n'est possible d'identifier le référent potentiel des éléments que le poète mentionne, comme s'il était habituel de trouver ces éléments hétéroclites assemblés dans un poème, ce qui va dans le sens d'une interprétation du présent comme l'expression de la répétition infinie de cette journée. Quelques articles définis se signalent par leur rareté : 《les arbres », « la tête », « le dos », « le dernier temps ». La fonction habituelle de l'article défini est soit de désigner quelque chose qui a été identifié auparavant dans la conversation ou le récit, soit de renvoyer à la classe de l'élément mentionné. Il est difficile de trancher entre ces deux options à la lecture de ce poème; le poète peut tout aussi bien parler des « arbres » comme de tous les arbres qui bordent évidemment tous les chemins imaginaires, ou tenter de créer un faux sentiment d'intimité : nul besoin d'identifier davantage cette " tête », le lecteur sait bien de quoi le poète parle, donnant ainsi les signes d'une fausse intimité dans la mesure où rien dans le poème ne permet d'identifier le porteur de cette "tête ». Néanmoins, dans ce cas précis, le lecteur assidu de Reverdy aura déjà souvent croisé « la tête » dans ses vers et l'aura vue mentionnée dans des contextes suggérant qu'elle désigne en fait, par métaphore, le soleil ${ }^{1}$. Non que ce soit pour autant la seule lecture qu'on puisse en faire, et l'incertitude de ce poème suggère bien que le choix de la référence est ouvert. Le lecteur, en reconnaissant ce motif, ferait donc de cette apparence d'intimité une intimité réellement partagée avec le poète. Quand auparavant la connaissance intime du poète par son lecteur était due à l'abondance de détails de nature intime (sur sa vie personnelle, psychologique, sur ses sentiments les plus profonds) dont le poète tissait ses vers, dans les poèmes de Reverdy, cette connaissance intime s'appuie sur une lecture assidue de l'œuvre du poète permettant d'élaborer une interprétation du poème.

Ce poème illustre un dernier point important de la manière poétique de Reverdy : l'absence de qualité du sujet énonciateur. L'utilisation du pronom «je » est très élusive dans les vers suivants; aucun indice ne donne d'information sur l'identité de la personne derrière ce « je ». La situation décrite : suivre une autre personne ou entité inconnue, peut être lue au sens propre ou figuré, selon le sens qu'on veut donner à ce « tu » (une personne ou l'inspiration par exemple). "Tu» peut même, dans ce cas particulier, renvoyer à la «tête » de la fin de la première laisse, dans la mesure où, si celle-ci renvoie potentiellement au soleil, c'est peut-être celui-ci que le poète cherche à suivre. Le vers « maintenant la tête a tout pris » peut renvoyer au moment où le soleil, disparaissant à l'horizon noie tout d'un reflet rouge ;

${ }^{1}$ Cf. Collot, $1981: 33$ et 45 (Tout le premier chapitre propose des analyses de la représentation de la figure paternelle en astre solaire, ainsi que sous divers autres figurations, et de l'agonie voire du meurtre qui parfois accompagne le coucher de soleil). 
" l'impossible repos » peut, dans la même veine, être une référence à la mort solaire que Reverdy décrit ailleurs dans ses poèmes, peignant parfois le coucher de soleil comme un meurtre. Les lectures possibles pour ce court poème sont multiples étant donné le vague de la référence ici érigé en règle. Le « je » est si dépourvu de toute qualité qu'il n'est même plus possible d'y attacher un genre : l'énonciation peut être prise en charge par un homme aussi bien qu'une femme. C'est ce qui rend possible la double lecture du poème : que le lecteur choisisse tantôt de passer derrière le « je » ou derrière le «tu ». Le portrait, pour ainsi dire, que le poète peint ici de luimême n'est pas sans rappeler les célèbres derniers vers de son poème testamentaire, Sable mouvant (Reverdy, 2010b : 1407 et ss.) :

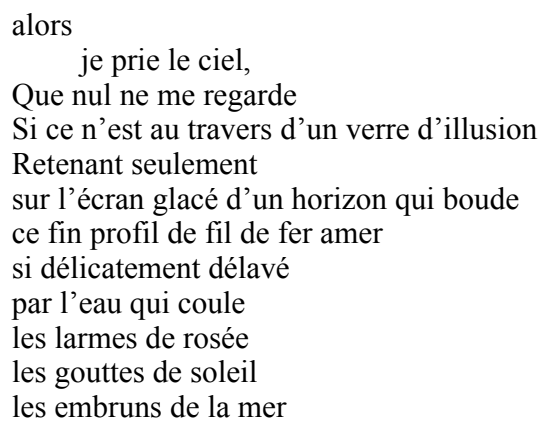

Ces derniers vers, l'adieu du poète à la poésie et ce faisant à ses lecteurs, rappelle son souhait de ne pas voir sa vie examinée. Le poète ne veut pas, en tant que personne, être traqué dans ses vers. A cette fin, il met plusieurs distances entre lui et son lecteur : il se laisse voir à travers un «verre d'illusion » et non directement, ce qui modifie déjà la vision que le lecteur peut avoir du poète. Plutôt que de laisser un portrait de face, il ne se montre que de profil, et celui-ci n'est constitué que de lignes en « fil de fer », non de surfaces; il est donc vide et le lecteur peut dessiner à l'intérieur pour le remplir, pour suivre la métaphore du portrait. Ces vers laissent également entendre que les lignes que le poète laissent de lui ne sont que les gros traits d'un profil, qu'il n'est pas vraiment là, il n'est pas présent entre les lignes et c'est au lecteur de suppléer ce qui manque là où Reverdy refuse de laisser voir son intimité. La façon dont le poète met en scène un «je » évidé de subjectivité révèle donc les signes d'une stratégie de déplacement et de redéfinition de l'intimité dans ses vers, de celle du poète à celle du lecteur.

L'usage que Reverdy fait du pronom « je » représente l'une des clefs possibles, comme Collot en a l'intuition dans La Matière-émotion, pour comprendre certaines des positions de Reverdy sur des composantes majeures de son œuvre, notamment le lyrisme et, ici en particulier, l'intimité. La représentation du pronom varie au fil des recueils, comme un relevé de ses occurrences peut le révéler. Ainsi, "je » domine les recueils Quelques poèmes (1916), La Lucarne ovale (1916), Les Ardoises du toit (1918), Les Jockeys camouflés (1918), et La Guitare endormie (1919). Cela n'empêche pas la création de ce que Collot qualifie d'une «allure imperson- 
nelle et "objective" » (Collot, 1997: 203). Les marques de première personne disparaissent progressivement dans ce recueil : le «je» ne représente plus que l'équivalent de la moitié des «on », étrangement, le pronom possessif de première personne est encore assez bien représenté. « Je » disparaît totalement dans Etoiles peintes (1921) et Coeur de chêne (1921) au profit du "on », réapparaît ensuite timidement dans Cravates de chanvre (1922), domine à nouveau Grande Nature (1925), avant d'être complètement chassé de La Balle au bond (1928). Sa réapparition dans Sources du vent (1929) ouvre une nouvelle période de représentation stable, avec Pierres blanches (1930), Ferrailles (1937) et Plein verre (1940). Le Chant des morts (1944-1948) et Bois vert (1946) marquent un nouveau tournant, le « je » y est représenté, mais les pronoms personnels y sont en général rares, présageant de leur quasi disparition dans Soleil au plafond (1955) ou de leur maigre représentation dans Liberté des mers (1960). Son recueil testamentaire, Sable mouvant (publié à titre posthume en 1966, composé en 1959) est l'occasion d'un retour $\mathrm{du}$ « je » décomplexé pour l'adieu final du poète à la poésie.

Ces fluctuations dans l'usage de la première personne chez Reverdy indiquent deux choses : d'une part, la façon dont il considère le « je » dans ses poèmes connaît une voire deux crises majeures durant sa carrière, qui ne seront pas considérées ici, dans la mesure où elles ne peuvent guère être plus que des objets de conjecture. D'autre part, cela suggère également que la source de l'émotion dans ses poèmes, ce dont il fait la fin de toute poésie, ne réside pas dans le «je » comme sujet de l'énonciation. Par voie de conséquence, la source du lyrisme se trouve relativement indépendante du « je ». Dans les deux cas, la création d'une intimité avec le lecteur ne peut procéder ni de l'exposition de l'intimité du sujet énonciateur, offerte au lecteur; ni d'une connaissance, moins profonde mais inscrite dans la durée, des circonstances du sujet énonciateur qui ferait du lecteur son intime. Une intimité est pourtant créée et appelée de ses vœux par le poète, quand les ressorts habituels qui servent à la construire sont absents de ses vers. Ce paradoxe apparent n'est pas sans rappeler celui qui entoure le lyrisme reverdien, à la fois rejeté et refondé, et dont l'élucidation permet de mieux comprendre la façon dont Reverdy peut viser l'intime à partir d'un quasi néant.

Deux possibilités émergent des poèmes de Reverdy, ainsi que de leur relecture par la critique. La première façon dont le poète peut toucher son lecteur au cœur, en ce qu'il a de plus intime consiste à mettre en scène ou à suggérer dans ses poèmes des scènes archétypales de la vie humaine. La partie «Le père » d'Horizon de Reverdy (Collot, 1981 : 13-43) est à ce sujet éclairant : l'ouvrage retrace à travers les poèmes de Reverdy les signes d'une mise en scène de la relation au père - habituellement représenté par l'astre solaire selon le critique - à travers ses différents stades problématiques : la surveillance ou censure paternelle, l'attente du père, le désir de castration et sa réalisation par le fils. Il est possible de proposer une relecture du poème «Et là » selon ces analyses; le soleil-père disparaîtrait à l'horizon, le «je », son fils, à l'évidence veut le suivre et hésite à le faire, se demandant s'il connaît vraiment son père absent, ou plutôt, à l'agonie, comme peuvent le suggérer les vers « une âme détachée s'amuse / et traîne encore un corps qui 
s'use ». Ainsi, face à ces thèmes considérés comme universels, le lecteur est encouragé à rechercher dans sa propre histoire intime de quoi alimenter la lecture et la compréhension du poème et combler les manques à l'aide d'éléments puisés en luimême. Une analyse de ce genre, bordant la psychologie, quoique utile et révélatrice de sens, n'est pas sans danger : elle pourrait encourager à faire une lecture unilatérale de ce poème quand d'autres sens peuvent également lui être donnés; elle peut représenter un philtre par lequel le lecteur pourrait chercher à faire passer tous les poèmes de Reverdy ; enfin, il n'est pas possible d'appliquer ce genre de raisonnement à l'ensemble de l'œuvre de Reverdy. Une étude serrée du lexique dans ses différents recueils révèlent que si le terme de «soleil» ou ses synonymes sont en effet très fréquents dans la première période de Reverdy, ils diminuent de façon importante après 1925, comme si le poète, pour peu qu'il ait effectivement voulu mettre en scène sa relation avec son père, avait trouvé un apaisement et était passé à autre chose. Cette manière de toucher le lecteur en son point le plus intime ne serait donc valable que pour la première partie de l'œuvre du poète et se trouve ainsi, de fait, invalidée.

L'autre manière dont Reverdy parvient à mettre en jeu ce qu'il y a de plus intime en l'homme repose sur la combinaison astucieuse de l'image poétique, du nouveau lyrisme que Reverdy cherche à créer et de ce « je » apparemment vide qu'il inscrit au cœur de ses poèmes. L'analyse de « Secret» du recueil Les Ardoises du toit et sa relecture par Collot permettra de voir la façon dont cette association s'effectue (Reverdy, 2010a : 1984).

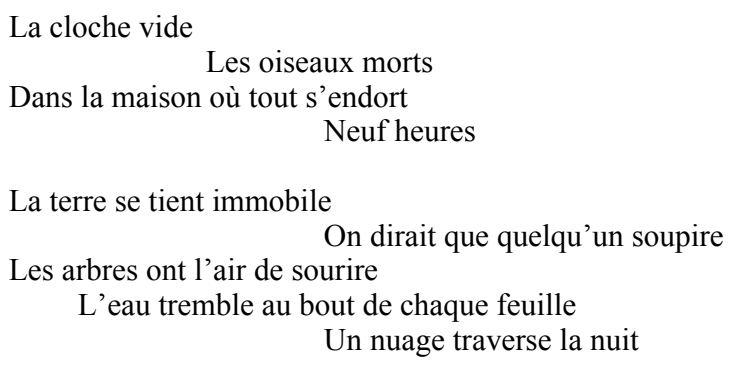

Devant la porte un homme chante

La fenêtre s'ouvre sans bruit

Collot fait apercevoir, dans l'analyse qu'il propose du poème, comment le lyrisme peut y naître sans l'ancrage dans une subjectivité. Il montre que si le poème est dépourvu de pronom personnel, à part un «on », l'énonciation semble se faire d'elle-même ; les images, le rythme et les sonorités convergent pour créer « une impression commune de manque ». Il conclut son analyse en déclarant :

Ainsi se trouve posée une " tonalité affective ", qui dominera tout le poème ; non pas un sentiment bien défini qu'on pourrait attribuer à un sujet précis, et qui s'exprimerait ainsi : « Je me sens 
seul ce soir, dans la maison où tout s'endort », mais un climat émotionnel lié à une certaine coloration du monde et à une certaine résonnance des mots (Collot, 1997 : 209).

Le poème progresse selon lui vers la création d'une tonalité affective dont l'expression est, selon les travaux de Rodriguez sur le sujet, le projet du lyrisme ${ }^{2}$. Or pour ce faire, nul besoin de sujet en charge de l'énonciation, il est porté par la réalité décrite dans le poème, ainsi que par la manière de le dire. Le lyrisme, selon Reverdy, naît en effet « de deux mots pour la première fois et avec justesse accouplés. Il jaillit d'une image inouïe, forte, inattendue, vraie, capable de placer une production nouvelle de l'esprit dans la réalité ». (Reverdy, 1968 : 558). C'est exactement ce qui a lieu dans le poème lorsqu'il nous présente cette "cloche vide »; « vide » est un adjectif impertinent pour une « cloche », dans la mesure où elle ne peut davantage être pleine, à moins de considérer que son battant la remplirait, ou plutôt, la rendrait complète. Cette " cloche vide » serait alors une cloche dépourvue de son élément essentiel, aussi morte que les « oiseaux » du vers suivant. On comprend que le poète suggère par là qu'elle ne peut faire de son, mais en utilisant à dessein un adjectif appartenant à un autre registre il déplace le sentiment de vacuité à l'ensemble du poème. L'image de la « terre [qui] se tient immobile » est tout aussi surprenant pour le lecteur dans la mesure où elle décrit une impossibilité physique en même temps qu'un sentiment de fin du monde. L'image des «arbres [qui] ont l'air de sourire» est plus commune pour la poésie de Reverdy, mais elle ne renvoie à rien dans la réalité, elle ne sert qu'à accentuer le sentiment de désolation qui règne dans le poème, comme si la solitude était si grande que la nature en venait à assumer des traits humains pour compenser. C'est de ce genre d'images que, selon la théorie poétique de Reverdy, naît le «lyrisme mouvant et émouvant de la réalité » (Reverdy, 2010b : 546) au cœur du poème, et non de la prise en charge de l'énonciation par un sujet. Rodriguez explique ainsi comment dans ce qu'il appelle « le pacte lyrique », le sujet peut disparaitre au profit de la « voix », comme c'est le cas dans « Secret» :

Dans le pacte lyrique, en effet, tout comme dans le pacte fabulant, le locuteur principal ne produit pas toujours un effet de sujet identifiable, qui pourrait notamment s'inscrire à la première personne. C'est pourquoi nous préférons détacher la voix, comme instance chargée de l'énonciation, du sujet qui engage précisément la dimension du personnage. Si dans une vision commune, une voix renvoie toujours à un sujet, il arrive fréquemment dans les textes littéraires qu'elle ne soit pas assimilable à une origine aussi individuée. La voix peut en effet apparaître comme l'origine de l'énonciation sans diriger par des repères minimaux une esquisse de vie subjective personnelle [...].

C'est pourquoi nous emploierons l'expression « voix lyrique » pour désigner l'instance virtuelle principale qui produit l'énonciation [...].

\footnotetext{
2 « Le pacte lyrique articule la mise en forme affective du pâtir humain. Son effet global consiste à faire sentir et ressentir des rapports affectifs au monde [...]. » (Rodriguez, 2003 : 94-95).
} 
Notre conception de la voix se détache d'un sujet parlant individué, elle la dépersonnalise [...]. Elle porte et organise le langage, tout en manifestant le degré de profération antérieur à l'acquisition de la parole (Rodriguez, 2003 : 142).

Il est donc possible de séparer « la voix » du sujet, tel que le fait couramment Reverdy, et d'ancrer ainsi l'expression lyrique dans quelque chose de plus profond, d' « antérieur à l'acquisition de la parole ». Le lyrisme parvient ainsi à mettre à jour ce qu'il y a de plus caché et de plus secret, et ce faisant de plus intime chez le poète. Celui-ci parvient ainsi à mettre en mot le degré le plus profond de l'expérience humaine : l'accord de la « relation sujet-monde, sa situation et son horizon avant toute action, toute saisie réflexive» (Rodriguez, 2003 : 107), ce qui représente la définition des «tonalités affectives » pour le critique. La poésie reverdienne vise ainsi par l'image à susciter le lyrisme, et par le lyrisme, à faire remonter l'intime à la surface, à créer un pont entre poète et lecteur. Le lecteur reçoit à son tour cette mise en mots d'un indicible en son intimité profonde, grâce à l'image. Elle est en charge, chez Reverdy, de créer un «choc» chez le lecteur, à la manière d'une épiphanie, et de représenter pour lui la mise en mots de quelque chose qu'il a toujours chercher à se dire, chose si intime, si propre à son expérience perçue comme unique du monde que les mots pour l'exprimer l'ont toujours laissé en défaut. La poésie de Reverdy vient offrir au lecteur les termes nécessaires pour mettre en mots son expérience par le biais de l'image. C'est ainsi que sa poésie peut se permettre d'être dépourvue de pronoms, ou de proposer un sujet émacié, évidé de toute qualité dans la mesure où il appelle le lecteur à remplir lui-même les vides laissés par sa propre expérience du monde.

L'intimité que propose Reverdy est donc étrangement impersonnelle. Il ne s'agit pas dans ses vers de devenir « intime ", au sens de "personnel » avec le poète qui mettrait en scène sa vie personnelle, ni de développer une connaissance intime de la manière poétique de Reverdy, mais plutôt de parvenir à l'intimité en ce qu'elle a de plus essentielle : l'humanité que partage chaque homme. Reverdy écrit une poésie qui se propose de dire l'indicible - l'être-au-monde —, pour que le lecteur puisse y reconnaître sa propre relation au monde que jusque là il ne pouvait que subir, faute de mots pour la penser. En mettant la subjectivité à l'arrière-plan, le poète met paradoxalement le sens de la communauté au premier plan d'une poésie assez vague dans ses références, et précise dans ses images, pour que tous puissent la prendre pour eux-mêmes. C'est en retirant le « je » privé de ses poèmes que Reverdy rapproche le plus sa poésie de l'homme. Ce faisant, il s'inscrit à la fois dans une tradition d'écriture de l'intime qui émerge avec le romantisme et s'en dégage, rendant cette fois possible une écriture non du soi mais de l'être.

\section{RÉFÉRENCES BIBLIOGRAPHIQUES}

Collot, M., (1981) Horizon de Reverdy. Paris, Presses de l'Ecole Normale Supérieure.

Collot, M., (1997) La Matière-émotion. Paris, Puf.

Hugo, V., (1967) Les Contemplations. Paris, Gallimard, Coll. Poésies. 
Proust, M., (1971) Contre Sainte-Beuve. Paris, Gallimard, Coll. Bibliothèque de la Pléiade.

Reverdy, P., (1968) Le Gant de crin. Paris, Flammarion.

Reverdy, P., (2010a) Euvres complètes. T. I. Paris, Flammarion.

Reverdy, P., (2010b) Euvres complètes. T. II. Paris, Flammarion.

Rodríguez, A., (2003) Le Pacte lyrique: configuration discursive et interaction affective. Sprimont, Pierre Mardaga. 